\title{
Characteristics of patients with shingles admitted to a district general hospital
}

\author{
Rosemary Morgan, Debra King
}

\begin{abstract}
Summary
Little is known about why some patients with shingles are admitted to hospital. We reviewed 72 case notes from a list of 80 patients admitted to hospital with shingles over a six-year period. Pain was the main complaint of the patients admitted, most of whom were elderly and lived alone. The commonest site of involvement in hospital admissions was the eye (herpes zoster ophthalmicus). Diagnosis of shingles was made after admission in 12 patients, eight of whom had originally been diagnosed as having an acute medical or surgical condition. We conclude that the prodromal phase of shingles may lead to misdiagnosis.
\end{abstract}

Keywords: shingles; herpes zoster ophthalmicus

Shingles is a common condition, ${ }^{1}$ the incidence of which increases with age. Although most patients with singles are seen by their general practitioner, a small number are admitted to hospital each year in the UK (RCGP Data, a weekly return service provided by the Birmingham Research Unit of the Royal College of General Practitioners). Since only a few are admitted to hospital, many hospital doctors are unfamiliar with the spectrum of disease that shingles can produce and its optimal management. In 1995, the British Society for the Study of Infection published guidelines on the management of shingles. ${ }^{2}$

While the reasons for admitting immunosuppressed patients with shingles to hospital for treatment are self-evident, very little is known about why other patients with shingles are admitted to hospital.

\section{Methods}

We undertook an audit of 72 case notes selected from a computerised list of 80 patients who had been admitted to hospital with a diagnosis of shingles over a six-year period. Eight patients who had been wrongly labelled as having shingles but had post-herpetic neuralgia (pain more than 30 days prior to admission) were excluded. Data collected included details of the presenting complaint at the time of admission, subsequent management, and follow-up arrangements. Analysis was performed using ARCUS.

\section{Results}

There were 50 women and 22 men, mean age 77 years (standard deviation 9.9, range 46-95 years). Most (48/72) lived alone. The reasons for admission are shown in the table. The median duration of shingles at the time of admission was four days (range 0-25 days).

Shingles had been diagnosed in 60 of the patients prior to admission; the diagnosis was made after admission in 12 patients. Four patients were initially thought to have acute abdominal conditions and were admitted to surgical wards, one with a diagnosis of acute appendicitis, and three with acute cholecystitis. Others had the following provisional diagnoses on admission: renal colic (1), pulmonary embolism (1) and ischaemic chest pain/ myocardial infarction (2). There was no clear diagnosis made in the remaining four patients.

Only two patients received parenteral acyclovir and both of these were immunosuppressed on chemotherapy. A total of 13 patients were on steroid therapy prior to developing shingles, including nine on inhaled steroids, all over the age of 70 years.

The site of involvement was given in all patients (box 1), but dermatomal distribution was documented in only 54 patients. Approximately $42 \%$ (30) of patients had herpes zoster ophthalmicus (eye involvement) and of these

Table Site $v s$ presenting complaint

\begin{tabular}{lcllll}
\hline Site & Pain & Nausea & Falls & Dizziness & Confusion \\
\hline Face & 2 & 1 & & 1 & \\
Ear & 1 & & & & \\
Neck & 1 & & & & 2 \\
Chest & 20 & 1 & 2 & 2 & 3 \\
Eye & 22 & 1 & & & \\
Abdomen & 11 & 1 & & \\
Buttock & 1 & & 2 & 3 & 5 \\
Total & 58 & 4 & 2 & \\
\hline
\end{tabular}

\begin{tabular}{|lr|}
\hline Site of shingles & \\
\hline Site & $n$ \\
- face (no eye involvement) & 3 \\
: ear (Ramsay Hunt syndrome) & 2 \\
- neck & 1 \\
- chest & 23 \\
- eye (Herpes zoster ophthalmicus) & 30 \\
- abdomen & 12 \\
buttock & 1 \\
\hline
\end{tabular}

Box 1 
12 were direct admissions under the consultant ophthalmologists.

A total of 58 patients received acyclovir (24 were on acyclovir prior to admission and 34 were started on acyclovir on/after admission). Of the 14 patients who did not receive acyclovir, five had had shingles for less than 72 hours at the time of admission. Conversely, of the 34 patients started on acyclovir on/after admission, nine had had shingles for four or more days prior to admission.

Topical acyclovir was given to 21 patients after admission of whom 12 had eye involvement and were under the care of an ophthalmologist. The majority of patients (70) did not receive a topical anaesthetic.

A variety of analgesia was prescribed (box 2 ). Some patients (35) received multiple drugs. Of patients admitted with pain (58) as the presenting complaint, four had no analgesia prescribed.

Eleven patients with eye involvement were not referred to an ophthalmologist for assessment. None of these patients had follow-up appointments. Only four patients were referred to a pain specialist, either on the ward or at the time of discharge.

The mean duration of hospital stay was 13 days (standard deviation 13.2, range 3-98 days). Less than $50 \%$ (29) of patients were given further hospital follow-up.

\section{Discussion}

Shingles (Herpes zoster), results from the reactivation of varicella zoster virus from latent sites in the dorsal root ganglia. ${ }^{1}$ It is usually a very painful condition and it is not surprising that pain was the major presenting complaint in most patients in this study. However, other patients with shingles who also experience pain are managed at home by their general practitioner. The reasons for hospital admission are probably multifactorial and include multiple symptoms causing disability and lack of social support which is particularly relevant in the elderly; this probably accounts for the older population in this study.

Pain is not a universal symptom in shingles. In some patients, particularly the young, shingles may occur without any discomfort or pain, conversely pain may occur without cutaneous rash (zoster sine herpete), and may account for some cases of atypical facial palsy or trigeminal neuralgia. ${ }^{3}{ }^{4}$ The majority of patients in this study were elderly (over 70 years of age) and most lived alone.

A prodrome that recurs in approximately two-thirds of patients with shingles involves a burning or painful sensation in the dermatomal skin supplied by the involved nerve, which on examination may appear entirely normal. It is this prodrome which may cause diagnostic difficulty, with erroneous diagnoses leading to acute medical/surgical admissions (box 3). A minority (16\%) of patients in this study developed overt shingles with its obvious rash after admission. Although 13 patients were on steroids prior to developing shingles, the size of

\begin{tabular}{|lr|}
\hline Pain relief & \\
\hline Analgesic & $n$ \\
- paracetamol & 5 \\
- NSAIDs & 6 \\
- co-proxamol & 15 \\
- co-codamol & 21 \\
- carbamazepine & 14 \\
- dihydrocodeine & 11 \\
- amitriptyline & 17 \\
\hline
\end{tabular}

Box 2

\begin{tabular}{|l|}
\hline Misdiagnoses with shingles \\
\hline - acute appendicitis \\
- acute cholecystitis \\
- pulmonary colic \\
- pneumonia \\
- myocardial infarction
\end{tabular}

Box 3

the study population is too small to draw any meaningful conclusions.

The commonest site of shingles is the trunk, being the largest surface area with the largest dermatomal supply (figure 1). ${ }^{56}$ In this study, however, the commonest site was the eye (42\%) (ophthalmic herpes zoster) (figure 2). The high incidence of in-patients with ophthalmic herpes zoster may reflect the readiness of general practitioners to refer patients with eye involvement to hospital. In addition, patients

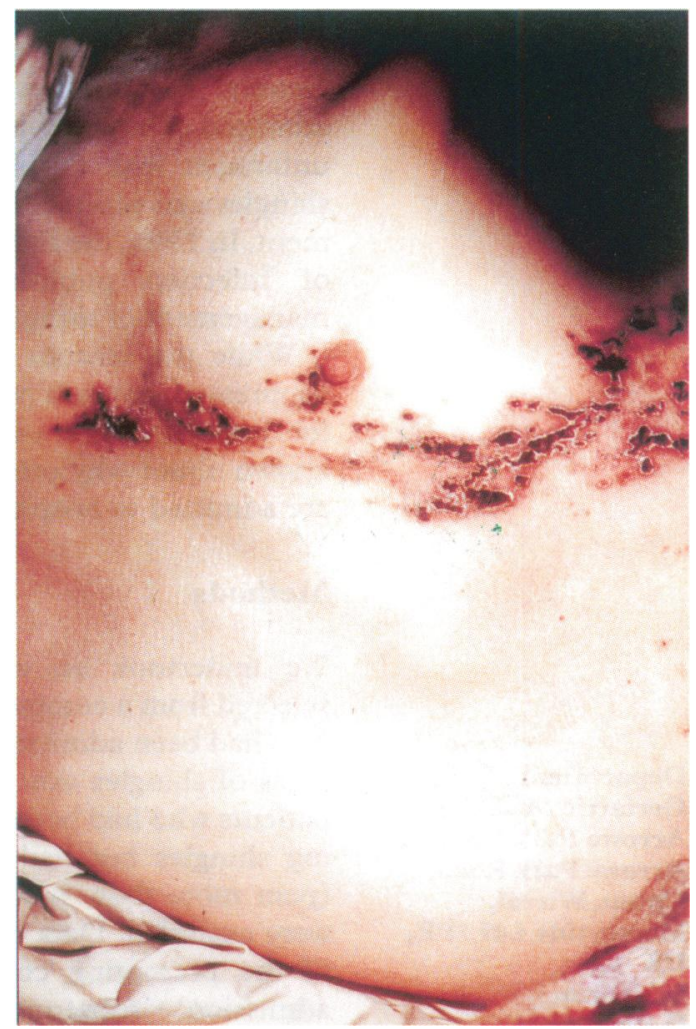

Figure 1 Shingles affecting thoracic dermatomal distribution 


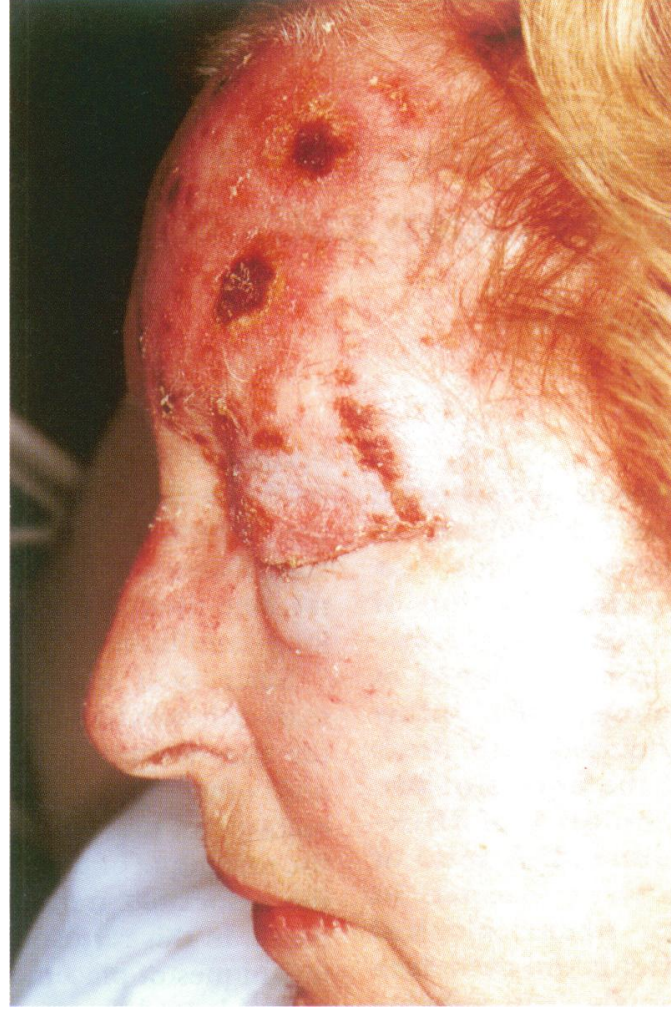

Figure 2 Ophthalmic herpes zoster

may themselves be more concerned about eye involvement and actively seek help or admission. Another possible explanation is that ophthalmic herpes zoster is a more painful condition, although there is no evidence for this. Since eye involvement may produce serious acute and chronic complications which threaten sight, an ophthalmic opinion is recommended. In this study, $37 \%$ (11) of patients admitted who had ophthalmic herpes zoster were not referred to the ophthalmologist for assessment.

There is an overall benefit from using acyclovir ointment in herpes zoster involving the ophthalmic division of the trigeminal nerve and in the prevention of ocular complications. ${ }^{7}$ Superficial punctate keratitis and dentritiform keratopathy are followed by fewer complications when treated by acyclovir ointment, possibly due to viral replication in the corneal epithelium. ${ }^{8}{ }^{9}$ In this study, none of the patients with eye involvement who had not been seen by an ophthalmologist received topical acyclovir.

There is considerable evidence that oral antiviral drugs commenced within 72 hours of the shingle rash appearing reduced the duration of pain, increased the rate of healing and

1 Hope-Simpson RE. The nature of Herpes zoster. A long term study and a new hypothesis. Proc $R$ Soc Med 1965;58:9. 2 Report of a Working Group of the British Society for the Report of a Working Group of the British Society for the study of Infection (BSSI). Guidelin

3 Rudra T. Zoster sine herpete. Br F Clin Pract 1990;44:284-5.

3 Rudra T. Zoster sine herpete. Br F Clin Pract 1990;44:284-5. the trigeminal nerve. Oral Surg Oral Med Oral Pathol 1993; 75:173-5.

5 Taylor-Robinson D, Caunt AE. Varicella virus. Vienna: Springer Verlag, 1972.

\section{Current guidelines on use of oral antiviral drugs in immunocompetent patients}

- over the age of 60 years presenting within 72 hours of rash

- consider also patients under this age who present with severe acute pain within 72 hours of rash

- patients of any age with ophthalmic involvement within the first 72 hours of rash

- patients with active zoster affecting the neck, limbs, and perineum, ie, cervical, lumbar and sacral dermatomes

Box 4

reduced ophthalmic complications. ${ }^{10}$ The current guidelines on oral antiviral drugs use are shown in box 4 . In this study the majority of patients $(80 \%)$ received acyclovir, although for some patients it was started too late to benefit. Unfortunately, five patients did not receive oral antiviral drugs despite onset of rash before 72 hours. There is a narrow therapeutic window of opportunity to influence the course of shingles and it should not be missed.

Some patients received carbamazepine (19\%) as part of pain relief, despite there being no evidence that carbamazepine has any effect on pain from acute shingles and is not without its side-effects.

\section{Conclusion}

Pain is the main presenting complaint of patients admitted to hospital with shingles. The commonest site of involvement for in-patients is the eye (ophthalmic herpes zoster). Unfortunately, not all patients with eye involvement were reviewed by an ophthalmologist, suggesting that increased education on shingles management is required. Patients who had an ophthalmic opinion were more likely to be on topical acyclovir and to have a follow-up appointment. The majority appear to receive simple analgesia only.

Only two patients had been referred to a pain clinic. This suggests either that most patients settled with simple analgesia or, alternatively, that further pain interventions were not considered.

For the hospital doctor, shingles is a rare condition and wider dissemination of the recent guidelines on its management ${ }^{2}$ is required, so they can be implemented for optimal management.

6 Juel-Jensen BE, MacCallum FO. Herpes simplex, varicella and zoster. London: Heinmann, 1972

7 Wagstaff AJ, Faulds D, Goa KL. Acyclovir, a reappraisal of its antiviral activity, pharmokinetic properties and therapeutic efficacy. Drugs 1994;47:153-205.

8 Lisegang TJ. Ophthalmic Herpes zoster. Diagnosis and antiviral therapy. Geriatrics 1991;46:64-71.

9 Hoang-Xuan T, Buchi Er, Herbort CP. Oral acyclovir for herpes ophthalmicus. Ophthalmology 1992;99:1062-71.

10 Bean B, Braun C, Balfour HH. Acyclovir therapy for acute herpes zoster. Lancet 1982:2:118-21. 